\title{
163. Treatment of Experimental Atherosclerosis by Pyridinolcarbamate
}

\author{
A Preliminary Report \\ By Takio Shimamoto, Tsutomu Fujita, \\ and Fujio Numano \\ Institute for Cardiovascular Diseases and Department of Medicine, \\ Tokyo Medical and Dental University \\ (Comm. by Tanemoto Furuhata, M.J.A., Oct. 12, 1965)
}

In the beginning of this century there were three incurable lesions having dead spaces with an accumulation of dead substances such as gumma of syphilis, caseous materials of tuberculosis and atheromatous substances of atherosclerosis. However the former two became curable since the discovery of modern chemotherapeutic agents and the atherosclerosis has been left for the solution of this problem.

Recently the author found a compound which antagonizes competitively and reversibly the venoconstrictive effect of bradykinin and lysylbradykinin in vitro and also in vivo. Using this compound we succeeded to show a definite curing effect in the atheroma of cholesterolfed rabbits.

Material and Method.

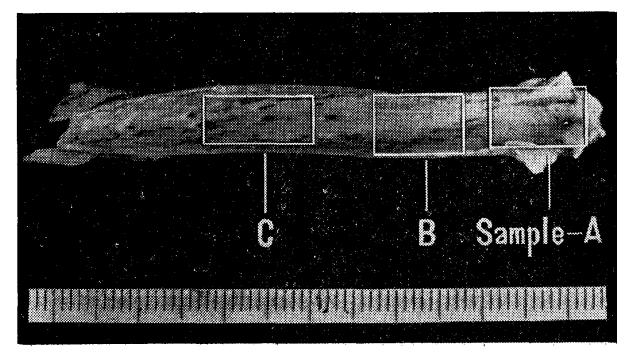

Fig. 1-A. The internal surface of rabbit's aorta of the control placebo group fed $1 \%$ cholesterol for 15 weeks. Microscopic finding of sample $B$ shows in Fig. 1-B. Twenty-eight albino rabbits from Takeda firm (6 females and 22 males) with starting body weight of $2.2 \pm 0.3 \mathrm{~kg}$ were utilized. In order to produce atherosclerosis ${ }^{5)}$ all animals were kept on pellets produced by Oriental East Co. containing 1 per cent of cholesterol for 15 weeks. These 28 atheromatous rabbits were divided into the placebo control group and the treated group. The treated group received daily pyridinolcarbamate in a dose of $10 \mathrm{mg} / \mathrm{kg}$ placed in a gelatin capsule by mouth and the placebo control group received potato starch placed in a gelatin capsule as a placebo.

Each 3 animals of both groups were sacrificed on the 4 th and 7 th day of the treatment respectively under the cholesterol feeding. In the remaining 16 animals the cholesterol pellet were discontinued after 15 weeks of the cholesterol feeding and they were kept on RC-5 pellets which contain no cholesterol. After $3,6,10$, and 15 


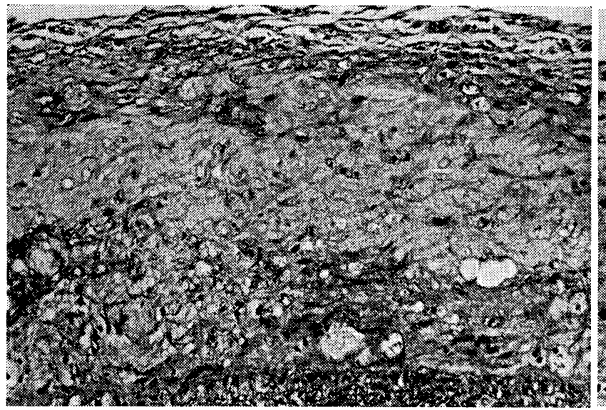

Fig. 1-B. (Sample-B of Fig. 1-A) H.E. Stain $\times 200$. Control rabbit fed $1 \%$ cholesterol pellet for 15 weeks. Note: Typical atheroma: Edema (upper part), acellular spaces with an accumulation of atheromatous material (middle) and degenerated foam cells (lower part). Almost no repair process is seen except a fibrosis in the innermost layer of the intima.

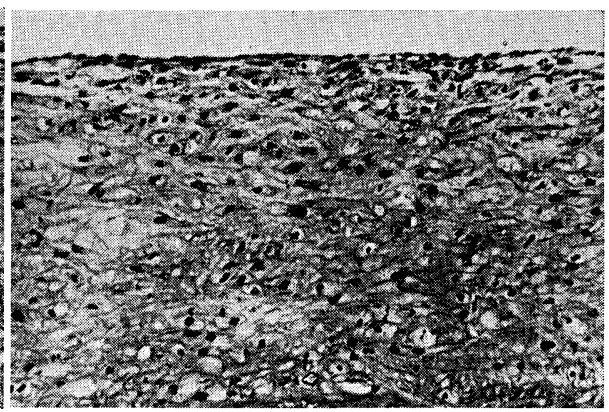

Fig. 2. H.E. Stain $\times 200$. Pyridinolcarbamate $(10 \mathrm{mg} / \mathrm{kg}$ p.o.) treatment was performed for 4 days in cholesterol-fed rabbits with severe atheroma under the continuous cholesterol feeding. Note: An appearance of fibroblasts, myoblasts (center) and some repair cells took place inside the hyalinous mass of atheroma, and this is a historical evidence ever reported. Disappearance of edema is also noted.

weeks of the treatment with pyridinolcarbamate or with placebo each 2 animals respectively were sacrificed.

In order to see the morphological changes including edematous changes of arterial walls, the rapid sampling and fixation of aortic specimen in ice-cold glutaraldehyde solution $(5 \%)$ or in one per cent osmium tetraoxide were used and the procedure was finished within 30 seconds after giving a stunning blow on the head of animals. For the embedding paraffin and also celloidin were used and hematoxylin-eosin stain, Elastica Van Gieson stain, Heidenhain's Azan variant, Sudan III and Sudan IV stain were used. For the identification of myoblast an electron microscopic technique was used.

Pyridinolcarbamate (Anginin) was supplied by Banyu Co. Ltd.

Results 1. Aortas of the animals of the placebo control group exhibited a severe atheromatous changes occupying over 50 per cent of the whole internal surface. The plaques seemed white and somewhat slightly yellowish and the lusterlessness of their surface was noted.

The histological findings: A typical feature of well known atheroma of cholesterol-fed rabbits was observed. The lesions exhibited some fibrous tendency in the superficial portion accompanied by marked edematous features as shown in Fig. 1-B.

In animals kept on cholesterol-free diet for 3 to 15 weeks minimal fibrous changes were seen inside the atheroma and also a few fibroblasts were found inside the atheroma and a few smooth muscle fibres appeared on the surface area of atheroma. However the amount of the fibroblasts and muscle fibres was small and the main parts of 
Table. I. Pyridinolcarbamate treatment in atherosclerosis of cholesterol-fed rabbits

\begin{tabular}{|c|c|c|c|c|}
\hline & Placebo & Control G & Group & Pyridinolcarbamate Group \\
\hline \multirow{2}{*}{$\begin{array}{l}\text { Histological } \\
\text { Findings in } \\
\text { At heromatous } \\
\text { Lesions }\end{array}$} & \multicolumn{3}{|c|}{ Treatment with Placebo for } & Treatment with Pyridinolcarbamate for \\
\hline & 4 d.s: 1 wks $^{*}$ & 3 wks 6 wks & 10 wks 15 wks & 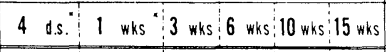 \\
\hline Hyalinous \& NecroticFoci & $4+4+4+$ & $+4+4$ & +++ \pm & $+- \pm---1------1$ \\
\hline Edema & $\omega *++++$ & $+4+ \pm$ & $+4+ \pm$ & + \pm++++++----- \\
\hline Foam Cells & D*⿻二丨 * * & $=\#$ & $+\omega+\cdots$ & $+\boldsymbol{+} \pm \pm+ \pm+ \pm \pm \pm \pm \pm \pm$ \\
\hline Collagen Fibres & \pm \pm \pm \pm \pm \pm & +++ & $-4+4$ & 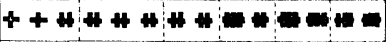 \\
\hline Elastic Fibres & $---1-\cdots$ & $\pm+t+$ & ++++ & $\pm \pm \pm++++++\infty+\omega=$ \\
\hline Smooth Muscle Cells & $---1--$ & \pm+++ & +++4 & $\pm \pm \pm-6+*+\infty-\infty \omega \infty$ \\
\hline
\end{tabular}

the atheroma were occupyed either by atheromatous mass showing severe fatty degeneration and necrotic foci or by an abundant accumulation of foam cells and the Sudan stain exhibited an abundant accumulation of sudanophilic substance occupying the whole atheromatous lesions (Fig. 5-B). The grade of stainable fat content seemed increased, not decreased, in animals, which were kept on the cholesterolfree basic feed especially for 6 to 15 weeks.

2. Aortas of treated animals. The aortas of animals treated with pyridinolcarbamate for 4 to 7 days under the cholesterol diet, exhibited grossly almost the same features as in the placebo control group. However, in the histological findings a striking difference was found. In animals received the compound for 4 days the atheroma exhibited already an appearance of myoblasts, fibroblasts and of fibrocytes inside the atheromatous mass as shown in Fig. 2 and an extracellular deposition of collagenous substances was already found though slightly. The edematous feature has already disappeared entirely and the foam cells exhibited a definite flattening.

In animals received the compound for one week a definite desposition of collagen fibres was found in atheromatous lesions. Young smooth muscle cells with their fibres, which are still fine and short and the elastic fibres appeared already in the middle part of atheromatous lesions.

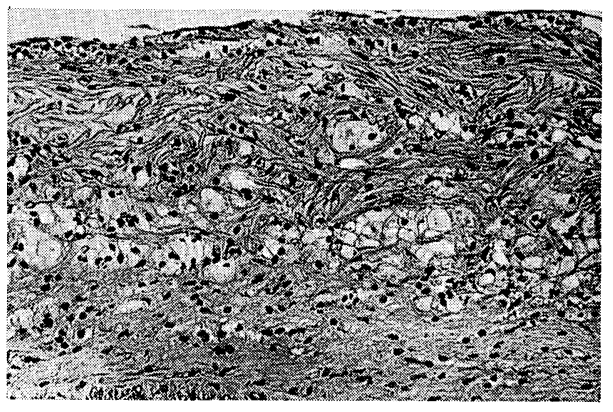

Fig. 3. H. E. Stain $\times 200$. Pyridinolcarbamate $(10 \mathrm{mg} / \mathrm{kg}$ p. o.) treatment was performed for 6 weeks in cholesterol-fed rabbits with severe atheroma (1\% cholesterol pellet was given for 15 weeks and all internal surface of aorta showed sudanophilic feature). Note: Regenerated smooth muscle fibres and elastic fibres separated the atheromatous mass into small pieces showing a definite curative effect of the compound. 
In animals received the compound for 3 and 6 weeks the surface of atheromatous plaque seemed somewhat luster. The most striking histological finding was the appearance of abundant mature smooth muscle fibres inside the atheroma, invading into the atheromatous mass occupying the hyalinous foci, and dividing the hyalinous substances or degenerated foam cells into smaller portions as shown in Fig. 3. The appearance of smooth muscle fibres was also accompanied by elastic fibres. In animals received the substance for 6 to 10 weeks the foam cells exhibited further degeneration and almost disappeared in the majority of the lesions except the atheroma locating on the point of bifurcation although showing definite degenerative features. The sudanophilic substance in the majority of the atheromatous lesions exhibited a definite reduction especially in the areas adjacent to medial layers.

In animals received the compound for 15 weeks the per cent surface involved by atheromatous lesions was definitely smaller in the

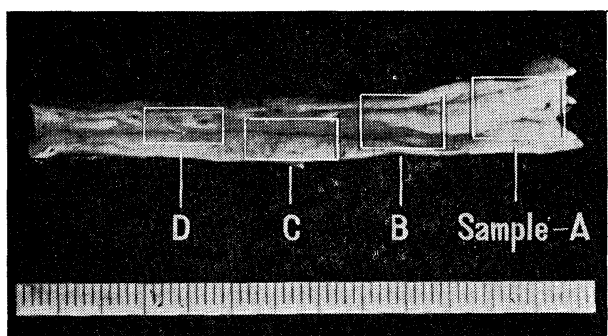

Fig. 4-A. The internal surface of aorta of the treated rabbit by pyridinolcarbamate $(10 \mathrm{mg} / \mathrm{kg})$ for 10 weeks after cholesterol feeding for 15 weeks. Microscopic finding of sample B shows in Fig. 4-B.

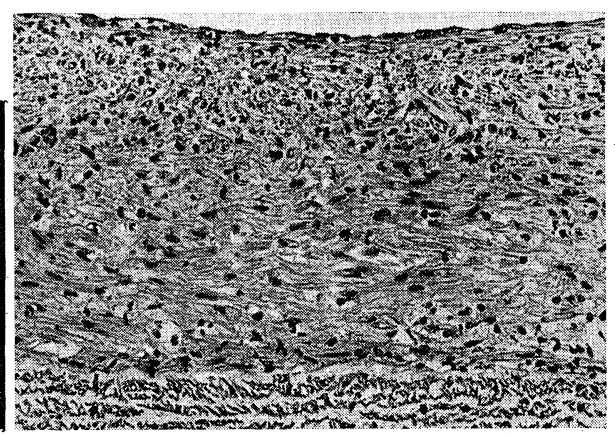

Fig. 4-B. (Sample B of Fig. 4-A) H.E. Stain $\times 200$. Pyridinolcarbamate $(10 \mathrm{mg} / \mathrm{kg})$ treatment was performed for 10 weeks in cholesterolfed rabbits with severe atheroma (1\% cholesterol pellet was given for 15 weeks). Note: The striking regeneration of smooth muscle fibres which seems to have replaced the atheromatous mass into the muscle fibres.

treated group as compared with the placebo control group.

In the histological findings the regenerated smooth muscle fibres seem to occupy almost fully the lesions in almost the half of the atheromatous lesions in the thoracic aorta and the elastic fibres were also found among the smooth muscle fibres as shown in Fig. 4-B. No foam cell was seen and the Sudan positive substance became minimal and scattered as small particles in such lesions. In the remaining lesions a few degenerated foam cells were seen divided into an extremely small spaces surrounded entirely by regenerated smooth muscle fibres. 


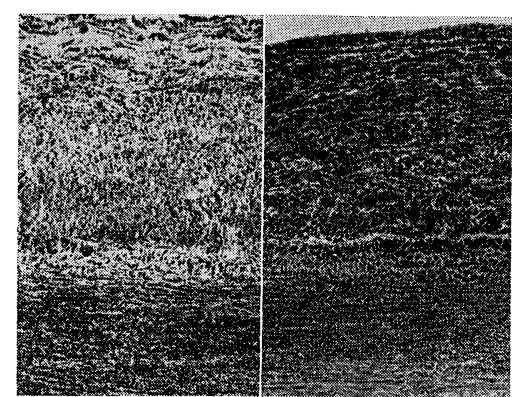

Fig. 5-A. $\times 100 . \quad$ Fig. 5-B. $\times 100$.

Sudan III stain of Atheromatous Lesions.

A: Treated by Pyridinolcarbamate for 10 weeks.

B: Treated by Placebo for 10 weeks.

Note: A marked reduction of sudanophilic substances was noted in atheromatous lesion of treated animals.

A sudanophilic substance was still found in these lesions, however it exhibited a striking further reduction as compared with that of the treated animals for shorter period (Fig. 5-A). There was no necrotic or hyalinous foci. At the beginning of this experiment the mean serum cholesterol level of test animals was $1651.3 \pm 68.5 \mathrm{mg} / \mathrm{dl}$ and $2,4,6,10$, and 15 weeks after the withdrawal of cholesterol diet it was still high amounting 1101, 651, 428, 191, and $113 \mathrm{mg} / \mathrm{dl}$ respectively. There was no significant difference between the control placebo and treated group.

Discussion. The early appearance of repair process in the atheroma was the most striking evidence in this treatment of experimental atherosclerosis with pyridinolcarbamate. It is well known that the repair process of common aseptic wound is fast and used to be finished within 8 to 10 days. In the repair process there are two processes; the production of fibrous material filling the dead spaces and the regeneration of local tissues. In the repair of atheromatous lesions the rapid and striking regeneration of smooth muscle fibres replacing the atheromatous mass is the most important finding in the treatment of atheroma with pyridinolcarbamate.

Since Virchow ${ }^{6}$ ) the appearance of edema, small round cells and of polymorphonuclear leucocytes has been described by many morphologist in atheromatous lesions ${ }^{7}$ and Rössle ${ }^{8)}$ proposed "his serous inflammation of artery" as a causative mechanism involved in atherogenesis. The authors ${ }^{9), 10)}$ found that so-called atherogenic substances such as cholesterol or adrenaline or high molecular weight substances induce the edematous arterial changes as an immediate reaction and the pretreatment of animals with bradykinin-forming enzyme inhibitor; trasylol or with bradykinin-antagonists such as pyridinolcarbamate or cyproheptadine or acetylsalicylate prevent the edematous reaction induced by these atherogenic substances. ${ }^{2)}$ These evidences obtained by the authors have indicated that kinin-induced venoconstriction $^{9,1)}$ in the vasa vasorum might possibly be the causative factor in the production of exudation in the arterial wall. Namely this compound may inhibit the excessive constriction of venous segments of vasa vasorum by bradykinin or by its active homologues 
which produce the exudation of arterial wall leading to the production, maintenance and progression of atheromatous changes and prevents the healing of atheromatous lesions.

The antagonistic effect of pyridinolcarbamate ${ }^{1), 2)}$ against the excessive venoconstriction by bradykinin might prevent the congestion and exudation and establish a smooth circulation in vasa vasorum which contributes to the healing process of the hitherto incurable atheroma by regeneration of local structural elements even under the high cholesterolemia. However, it is still premature to interprete the curative effect in that way.

Summary. Pyridinolcarbamate, a bradykinin-antagonist showed no hypocholesterolemic effect, but, exhibited definitely a curative effect in atheroma of cholesterol-fed rabbits. The appearance of its curative effect was prompt and the leading repair process was the replacement of atheromatous mass with regenerated smooth muscle fibres which begins early within the first week of the treatment and progresses gradually in the course of over 15 weeks even under the high serum cholesterol level.

Acknowledgement. The authors are grateful to Prof. Gonpachi Yajima and Assistant Prof. Shigeru Hatakeyama for their discussion and help in these studies. This work was supported by grants from NIH HE-05196 of U.S.A. and from the Ministry of Education of Japan. Pyridinolcarbamate was supplied by courtesy of Banyu Co. Ltd.

\section{References}

1) Shimamoto, T., Maezawa, H., Yamazaki, H., Atsumi, T., Fujita, T., Ishioka, T., and Sunaga, T.: Am. Heart J. (in press).

2) Shimamoto, T.: Meth. Acievm. exp. Path., Vol. 1, 1965 (in press).

3) Rowley, D. A.: Brit. J. Exp. Path., 45, 56 (1964).

4) Rocha e Silva, M.: Ann. N. Y. Acad. Sci., 105, 899 (1964).

5) Shimamoto, T., Fujita, T., and Numano, F.: Am. Heart J. (in press).

6) Virchow, R.: Die cellular Pathologie, in ihre Begründung auf physiologische und pathologische Gewebelehre, Berlin (1871).

7) McGill, H. C., Geer, J. C., and Strong, J. P.: Atherosclerosis and its Origin. (ed. by Sandler, M., and Bourne, G. H.) Academic press, New York and London, pp. 48 49 (1963).

8) Rössle, R.: Verhandl. Deut. Pathol. Ges., 27, 152 (1934).

9) Shimamoto, T., and Sunaga, T.: Japanese Heart J., 3, 521 (1962).

10) Shimamoto, T.: J. Atheroscler. Res., 3, 87 (1963).

11) Shimamoto, T., and Atsumi, T.: Japanese Heart J., 6, 57 (1965).

12) Shimamoto, T.: Jap. Med., 4, 230 (1965). 